Series A

I. MATHEMATICA

DISSERTATIONES

20

\title{
TWO AXIOMS OF SET THEORY WITH APPLICATIONS TO LOGIC
}

JOUKO VÄÄNÄNEN

HELSINKI 1978

SUOMALAINEN TIEDEAKATEMIA

https://doi.org/10.5186/aasfmd.1978.020 
Copyright (C) 1978 by Academia Scientiarum Fennica

ISSN 0355-0087

ISBN 951-41-0334-3

Received 24 July 1978

HELSINGIN KESKUSKIRJAPAINO OY HELSINKI 1978 


\section{Acknowledgements}

This paper is based on Sections 2.6 and 5.3 of my Ph.D. thesis (Manchester, 1977). I wish to use this opportunity to express my gratitude to my supervisor Dr. P.H.G. Aczel for the help and encouragement he provided during the preparation of the thesis.

The research for the thesis and the preparation of this paper were financially supported by the Osk. Huttunen Foundation.

Vantaa, July 1978

JOUKO VÄÄNÄNEN 


\section{Contents}

Introduction

Notation

1. The basic independence results 7

2. The consistency of $\sim(\mathrm{R}) \quad 10$

3. A connection with generalized quantifiers 12

4. Consistency results concerning (R1) and (R2) 15

$\begin{array}{ll}\text { References } & 18\end{array}$ 


\section{Introduction}

Consider the following predicates of set theory:

$$
\begin{aligned}
& \operatorname{Cd}(x) \leftrightarrow x \text { is a cardinal, } \\
& \operatorname{Rg}(x) \leftrightarrow x \text { is a regular cardinal, } \\
& \operatorname{Pw}(x, y) \leftrightarrow y \text { is the powerset of } x .
\end{aligned}
$$

These are all examples of $\Pi_{1}$-predicates and it is trivial that $\mathrm{Cd}$ is $\Sigma_{1}(\mathrm{Rg})$ and $\mathrm{Rg}$ is $\Sigma_{1}(\mathrm{Pw})$. In this paper we shall study the following two axioms:

(P) Pw is $\Sigma_{1}(\mathrm{Cd})$,

(R) Rg is $\Sigma_{1}(\mathrm{Cd})$.

Clearly $(P) \rightarrow(R)$. In Chapter 1 we consider various models of set theory in which $(P)$ and $(R)$ hold and also discuss models in which $(R)$ holds but $(P)$ is false. In Chapter 2 a model is constructed in which (R) is false.

Let LI be the logic with the Härtig-quantifier

$$
\text { I } x \text { y } A(x) B(y) \leftrightarrow \operatorname{card}(A)=\operatorname{card}(B),
$$

and $L^{I I}$ the usual second order logic. By means of the $\Delta$-operation of abstract logic, axiom $(P)$ can be characterized in terms of LI and I II $^{\text {: }}$ 


$$
(P) \leftrightarrow \Delta(L I)=\Delta\left(L^{I I}\right)
$$

If the quantifier

$$
\begin{aligned}
\mathrm{R} x \mathrm{yA}(\mathrm{x}, \mathrm{y}) \leftrightarrow & \{(\mathrm{a}, \mathrm{b}) \mid \mathrm{A}(\mathrm{a}, \mathrm{b})\} \text { has the order type } \\
& \text { of a regular cardinal }
\end{aligned}
$$

is used, a similar characterization obtains for $(R)$ :

$$
(R) \leftrightarrow \Delta(L I)=\Delta(L R)
$$

Thus the consistency results of Chapters 1 and 2 give also information of the relative strengths of the logics LI, LR and $\mathrm{L}^{I I}$. Consideration of these logics leads to some natural weakenings of $(P)$ and (R). These are discussed in Chapters 3 and 4 . In particular, a model is constructed in which the Löwenheim number of LI is strictly less than the Löwenheim number of LR.

\section{Notation}

Our notation is standard and follows in set theory mostly [2] and in abstract model theory [9]. Our metatheory is ZFC. The symbols $k$ and $\lambda$ refer to cardinals. The cardinal number of a set $x$ is denoted by $\operatorname{card}(x)$. The symbols $\alpha, \beta, \gamma$ and $\delta$ refer to ordinals. $P(x)$ is the powerset of $x$ and $\exp (k)$ is $\operatorname{card}(\mathcal{P}(\kappa))$.

The decision problem of a logic $L$ is the set of valid Lsentences. The Löwenheim number of $L$ is the least $k$ such that if $\varphi \in \mathrm{L}$ has a model, then $\varphi$ has a model of power $\leq \kappa$. The Hanf number of $L$ is the least $k$ such that if $\varphi \in L$ has a model of power $\geq k$, then $\varphi$ has arbitrarily large models. 


\section{The basic independence results}

1.1. In this chapter we show that (P) holds in such inner models as $L, L\left[O^{\#}\right]$ and $L[\mu]$ but fails to hold in models with generic reals. This also shows that $(R)$ does not imply $(P)$. The chapter ends with some results to the effect that the consistency of $\sim(R)$ is essentially harder to obtain than that of $\sim(P)$.

1.2. $\mathrm{P} r \circ \mathrm{p} \circ \mathrm{s} \mathrm{i} \mathrm{i} \circ \mathrm{n}$. Suppose the set a is $\Sigma_{1}(\mathrm{Cd})-$ definable and $\mathrm{V}=\mathrm{L}[\mathrm{a}]$. Then $(\mathrm{P})$ holds.

Proof. Let $k=\sup \left(\omega_{0}, \operatorname{card}(\mathrm{TC}(\{a\}))\right)$. Note that $k$ is $\Sigma_{1}(\mathrm{Cd})-$ definable. Suppose $\lambda>k$. We prove at first that $P(\lambda) \subset L_{\lambda}+[a]$. Suppose therefore $b \subset \lambda$. Let $M \prec L[a]$ such that $T C(\{a\}), b \in M$ and $\operatorname{card}(M)=\lambda$ (strictly speaking $M \prec L_{\alpha}[a]$ for a suitable $\alpha$ ). Let $\pi: M \cong L_{\alpha}[a]$ be the collapsing isomorphism, $\alpha<\lambda^{+}$. Now $\pi(b)=$ $\{\pi(\beta) \mid \beta \in b\}=\{\beta \mid \beta \in b\}=b$. Therefore $b \in L_{\alpha}[a] \subset L_{\lambda}+[a]$. We have proved $\mathcal{P}(\lambda) \subset \mathrm{L}_{\lambda}+[\mathrm{a}]$. Note that the predicate $x=L_{\lambda}+[a]$ is a $\Sigma_{1}(C d)$-predicate of a and $\lambda$. Now we have the following $\Sigma_{1}(C d)-$ definition of $\mathrm{Pw}$ :

$$
\begin{aligned}
& \mathrm{Pw}(\mathrm{x}, \mathrm{y}) \leftrightarrow \exists \lambda>\kappa \exists f \exists z \exists \alpha\left(\mathrm{L}_{\lambda}+[\mathrm{a}] \models \operatorname{Pw}(\alpha, z) \wedge\right. \\
& \mathrm{f}:\langle\mathrm{x}, \mathrm{y}, \varepsilon>\cong<\alpha, \mathrm{z}, \varepsilon>\wedge \alpha<\lambda) \text {. } \\
& \text { 1.3. } \mathrm{C} \circ \mathrm{r} \circ \mathrm{I} I \text { a } \mathrm{r} \mathrm{y} \text {. If } \mathrm{V}=\mathrm{L} \text { or } \mathrm{V}=\mathrm{L}\left[\mathrm{O}^{\#}\right] \text {, then }(\mathrm{P})
\end{aligned}
$$
holds. of $0^{\#}$ :

Proof. It suffices to recall the following $\Sigma_{1}(\mathrm{Cd})$-definition

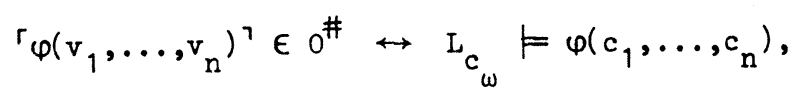

where $c_{1}, \ldots, c_{n}, \ldots, c_{\omega}$ are the first $\omega+1$ uncountable cardinals.

1.4. $\mathrm{L}$ e $\mathrm{m} \mathrm{m} \mathrm{a.} \mathrm{Suppose} \mathrm{V}=\mathrm{L}[\mu]$, where $\mu$ is a normal measure on a measurable cardinal. Then $\mu$ is $\Sigma_{1}(\mathrm{Cd})$-definable.

Proof. Suppose $\rho$ is the underlying cardinal of $\mu$. We prove at first that if $L_{\sigma}[u] \vDash " v$ is a normal measure on $\lambda$ ", where $\sigma$ and $\lambda$ are cardinals such that $L_{\sigma}[u]$ reflects $C d$, then $\lambda=\rho$ and 
$u=\mu$. Suppose $a \subset \lambda$ and $a \in L[U]$. Let $M \prec L[U]$ such that $T C(\{U\}), a \in M$ and $\operatorname{card}(M)<\sigma$. Let $\pi: M \cong L_{\alpha}\left[U^{\prime}\right]$ be the collapsing isomorphism. Note that $\pi(v)=\{\pi(x) \mid x \in u\}=$ $\{\{\pi(\beta) \mid \beta \in \mathbf{x}\} \mid \mathbf{x} \in U\}=\{\mathbf{x} \mid \mathbf{x} \in U\}=u$, and hence $u=u^{\prime}$. Also $\pi(a)=a$. As $\alpha<\sigma, a \in L_{\alpha}[u] \subset L_{\sigma}[u]$. Now it follows easily that $L[u] \models " u$ is a normal measure on $\lambda "$. By [6], $\rho$ is the smallest ordinal which is measurable in an inner model. Therefore $\rho \leq \lambda$. Suppose $\rho<\lambda$. By 6.7 and 5.8 of [6], UIt $t_{\lambda}(L[\psi], \mu)=L[U]$. It is well-known that $\rho^{+}<i_{01}(\rho)<\rho^{++}$, whence the cardinal $i_{01}(\rho)$ of Ult $t_{1}(L[\mu], \mu)$ is not a real cardinal. Note that $\lambda$ is a limit cardinal in $L[U]$ and hence also in $V$. Therefore $i_{01}(p)<\lambda$. Hence $i_{01}(p)$ is not a cardinal of $\mathrm{Ult}_{\lambda}(L[\mu], \mu)$. Let $f$ be a mapping which collapses $i_{01}(\rho)$ in $U 1 t_{\lambda}(L[\mu], \mu)$. But

$$
\mathcal{P}\left(i_{01}(\rho)\right) \cap U 1 t_{1}(L[\mu], \mu)=P\left(i_{01}(\rho)\right) \cap U 1 t_{\lambda}(L[\mu], \mu),
$$

and $f$ is coded by a subset of $i_{01}(\rho)$. This contradiction shows that $\rho=\lambda$. Now $v=\mu$ follows from the uniqueness of $\mu$ (see [6]). The following $\Sigma_{1}(\mathrm{Cd})$-definition of $\mu$ obtains:

$x \in \mu \leftrightarrow \exists \cup \exists \sigma \exists \lambda\left(L_{\sigma}[U] \models " v\right.$ is a normal measure on $\lambda " \wedge$ $\operatorname{Cd}(\lambda) \wedge \operatorname{Cd}(\sigma) \wedge \lambda<\sigma \wedge \forall \alpha<\sigma(\operatorname{Cd}(\alpha) \leftrightarrow$ $\left.\left.\mathrm{L}_{\sigma}[u] \vDash \mathrm{Cd}(\alpha)\right) \wedge \mathrm{x} \in \mathrm{u}\right)$.

1.5. C $\circ \mathrm{r} \circ \mathrm{l} 1 \mathrm{ary}$. If $\mathrm{V}=\mathrm{L}[\mu]$, where $\mu$ is a normal measure on a measurable cardinal, then ( $P$ ) holds.

1.6. The above results show that $(P)$ is consistent with ZFC if ZF is consistent. Corollary 1.5 shows that $(P)$ is also consistent with a measurable cardinal. It remains an open problem whether $(P)$ is (relatively) consistent with a supercompact cardinal. We turn now to the problem of independence of $(P)$ and $(R)$. In view of 1.2 , a natural candidate for a model of $\sim(P)$ is $L[a]$, where $a$ is a generic real:

1.7. P $r \circ p \circ s i t i \circ n$. Suppose $M$ is a countable model of ZFC and a is a real which is Cohen-generic over $M$. Then $\mathrm{M}[\mathrm{a}] \models \sim(\mathrm{P})$. 
Proof. Let $P$ be the set of Cohen-conditions. It is wellknown that $P$ is homogeneous. Hence, if $M[a]=(P)$, then $P \mid \vdash(P)$. Moreover, there is a single $\Sigma_{1}(\mathrm{Cd})$-formula $\varphi(\mathrm{x}, \mathrm{y})$ such that

$$
\mathrm{P} \quad \mid-\forall \mathrm{x} y(\mathrm{Pw}(\mathrm{x}, \mathrm{y}) \leftrightarrow \varphi(\mathrm{x}, \mathrm{y}))
$$

Let $b=\{n<\omega \mid 2 n \in a\}$. Then $b$ is $P$-generic over $M$ and $b \in M[a]$ but $a \notin M[b]$. Let $c$ be $\mathcal{P}(\omega)$ in $M[b]$. Then $M[b] \vDash \varphi(\omega, c)$. As $M[b]$ and $M[a]$ have the same cardinals, $M[a] \vDash \varphi(\omega, c)$, that is, $M[a] \vDash c=P(\omega)$. But this is absurd because $M[a] \vDash a \in c$ and $c \in M[b]$, but $a \notin M[b]$. This proves that $M[\mathrm{a}] \models \sim(\mathrm{P})$.

1.8. $\mathrm{C} \circ \mathrm{r} \circ \mathrm{I}$ I a $\mathrm{r} \mathrm{y}$. If $\mathrm{Con}(\mathrm{ZF})$, then $\mathrm{Con}(\mathrm{ZFC}+\mathrm{GCH}+\sim(\mathrm{P}))$ and $\operatorname{Con}(\mathrm{ZFC}+\sim \mathrm{GCH}+\sim(\mathrm{P}))$.

Proof. For the first claim, choose $M$ in 1.7 such that $\mathrm{M} \models \mathrm{GCH}$. For the second claim, choose $\mathrm{M}$ such that $\mathrm{M} \models \sim \mathrm{GCH}$. Adding one generic real does not affect $\mathrm{GCH}$ or $\sim \mathrm{GCH}$.

1.9. P $\mathrm{r} \circ \mathrm{b} I \mathrm{e} \mathrm{m}$. Does Con(ZF) imply Con $(\mathrm{ZFC}+\sim \mathrm{GCH}+(\mathrm{P}))$ ?

1.10. The situation with $(R)$ is more subtle. We shall show that no generic extension of $L$ can satisfy $\sim(R)$. The proof is based on R. Jensen's "Marginalia".

1.11. $\mathrm{P} r \circ \mathrm{p} \circ \mathrm{s} i \mathrm{t} i \circ \mathrm{n}$. If $\sim(\mathrm{R})$, then $\mathrm{O}^{\#}$ exists. Hence $(R)$ is true in every generic extension of a model of ZFL.

Proof. R. Jensen proves in [1]: If there is a singular limit cardinal which is regular in $\mathrm{L}$, then $0^{\#}$ exists. Thus if $0^{\#}$ does not exist, then a limit cardinal is regular if and only if it is regular in $\mathrm{L}$, that is,

$$
\begin{gathered}
\operatorname{Rg}(\kappa) \leftrightarrow \exists \lambda\left(\kappa=\lambda^{+} \vee(\kappa \text { is a limit cardinal } \wedge\right. \\
\left.\left.\mathrm{L}_{\kappa^{+}} \models \operatorname{Rg}(\kappa)\right)\right),
\end{gathered}
$$

which gives a $\Sigma_{1}(C d)$-definition for $\mathrm{Rg}$.

1.12. $\mathrm{C} \circ \mathrm{r} \circ \mathrm{I} I \mathrm{a} \mathrm{r} \mathrm{y}$. If $\mathrm{Con}(\mathrm{ZF})$, then $\mathrm{Con}(\mathrm{ZFC}+\mathrm{GCH}+\sim(\mathrm{P})$ $+(R))$ and $\operatorname{Con}(\mathrm{ZFC}+\sim \mathrm{GCH}+\sim(P)+(R))$.

1.13. The axiom $(R)$ is only interesting in the presence of large cardinals. In fact, if there are no weakly inaccessible cardinals, then 


$$
\operatorname{Rg}(\kappa) \leftrightarrow \exists \lambda\left(\kappa=\lambda^{+}\right)
$$

whence $(R)$ holds. Thus $\sim(R)$ implies the existence of at least one weakly inaccessible cardinal, and by 1.11, furthermore, of a proper class of weakly compact cardinals in $L$ (even in $L_{\omega_{1}}$ ). Therefore: 1.14. C $\circ \mathrm{r} \circ 1 \mathrm{I}$ a $\mathrm{y}$. Let $\mathrm{T}=\mathrm{ZFC}+$ there is a proper class of weakly compact cardinals. If $\mathrm{T}$ is consistent, then one cannot prove in $\mathrm{T}$ that Con $(\mathrm{T})$ implies $\operatorname{Con}(\mathrm{ZFC}+\sim(\mathrm{R}))$.

1.15. In contrast to 1.14 we shall prove shortly that Con(ZFC + there are uncountably many measurable cardinals) does imply Con(ZFC + $\sim(R)$ ).

\section{The consistency of $\sim(R)$}

2.1. In this chapter we construct a Boolean extension in which $\sim(R)$ holds. Our starting point is a model with uncountably many measurable cardinals, and we use an elaboration (essentially due to Magidor) of the so called Prikry-forcing to find the required Boolean extension. In view of 1.14 it seems legitimate to base the construction on large cardinals, although it is not clear whether many measurables are really needed.

2.2. L e m m a. Suppose $\left\{\rho_{\alpha} \mid \alpha<\omega_{1}\right\}$ is a sequence of measurable cardinals such that $\rho_{\alpha}<\rho_{\beta}$ whenever $\alpha<\beta<\omega_{1}$. There is a sequence $\left\{B_{\alpha} \mid \alpha<\omega_{1}\right\}$ of complete Boolean algebras such that the following hold for any $\alpha<\omega_{1}$ :

(1) $B_{\alpha}$ is a complete subaigebra of $B_{B}$ for $\alpha<B$.

(2) $\mathrm{B}_{\alpha}$ is homogeneous and preserves cardinals.

(3) $\mathrm{V}_{\alpha}^{\mathrm{B}}=\exp (\tilde{\mathrm{K}})=(\exp (\mathrm{k}))^{-}$for any cardinal $k$.

(4) $\mathrm{v}_{\alpha}^{\mathrm{B}}=\operatorname{cf}\left(\check{\rho}_{\beta}\right)=\check{\omega}$ for $B<\alpha$.

(5) $\quad v_{\alpha}^{B} \neq \check{\rho}_{B}$ is measurable for $\beta \geq \alpha$.

Proof. Let $P_{\alpha}$ consist of all sequences $\left\{<p_{B}, A_{B}>\mid \beta<\alpha\right\}$ such that $p_{B}$ is a finite sequence of elements of $o_{B}, p_{B}$ is empty but for finitely many $B<\alpha$, the set $A_{B}$ is in $D_{B}$ and finally $\cap A_{B}>U p_{B}$, 
where $D_{\beta}$ is a normal ultrafilter on $P_{\beta} \cdot P_{\alpha}$ is partially ordered as follows:

$$
\left\{<\mathrm{p}_{B}, \mathrm{~A}_{\beta}>\mid \beta<\alpha\right\} \leq_{\mathrm{P}_{\alpha}}\left\{<\mathrm{q}_{B}, \mathrm{~B}_{\beta}>\mid \beta<\alpha\right\}
$$

if and only if for all $B<\alpha, p_{B}$ extends $q_{B}, A_{B} \subset B_{B}$ and $p_{B}-q_{B} \subset B_{B}$. This notion of forcing is a simplification of the cne used in [8]. The full power of Magidor's forcing is not needed, mainly because we have no measurable limits of measurable cardinals. The advantage of the simplification is that the homogeneity is fairly easy to prove, whereas the homogeneity of Magidor's forcing is open (to the author's knowledge). Let $B_{\alpha}=R O\left(P_{\alpha}\right)$. Proofs of $(1)-(5)$ can be found in [8] and [14].

2.3. T is e or e $\mathrm{m}$. Suppose there are uncountably many measurable cardinais. Then there is a complete Boolean algebra $B$ such that $B$ preserves cardinais and $\exp (\alpha)$, and $v^{B} \models \sim(R)$.

Proof. Suppose $\left\{0_{\alpha} \mid \alpha<\omega_{1}\right\}$ and $\left\{B_{\alpha} \mid \alpha<\omega_{1}\right\}$ are as in 2.2. We claim that $v_{\alpha}^{B} \models \sim(R)$ for some $\alpha<\omega_{1}$. Suppose the contrary. Then for every $\alpha<\omega_{1}$, $\|(R)\|_{\alpha}^{B}>0$. Let $\alpha<\omega_{1}$. As $B_{\alpha}$ is homogeneous, there is a $\Sigma_{1}(\mathrm{Cd})$-formula $\varphi_{\alpha}(\mathrm{x})$ such that

$$
\mathrm{v}_{\alpha}^{\mathrm{B}} \vDash \forall \mathrm{x}\left(\operatorname{Rg}(\mathrm{x}) \leftrightarrow \varphi_{\alpha}(\mathrm{x})\right)
$$

As the number of all $\Sigma_{1}(C d)$-formulae is $\omega_{0}$, there are $\alpha$ and $B$ such that $\alpha<B<\omega_{1}$ and $\varphi_{\alpha}(\mathrm{x})=\varphi_{\beta}(\mathrm{x})$. By 2.2(5), $v_{\alpha}^{B_{\alpha}} \vDash \varphi_{\alpha}\left(\check{\rho}_{\alpha}\right)$. As $B_{\alpha}$ and $B_{\beta}$ preserve cardinals, $v^{B} B \models \varphi_{\alpha}\left(\check{\rho}_{\alpha}\right)$, and hence $v^{B} \vDash \operatorname{Rg}\left(\check{\rho}_{\alpha}\right)$, which contradicts $2.2(4)$.

2.4. C $\circ \mathrm{r} \circ 11$ a $\mathrm{r}$. Let $\omega_{1}-\mathrm{MC}$ be the statement "There are uncountably many measurable cardinals". If $\mathrm{Con}\left(\mathrm{ZFC}+\omega_{1}-\mathrm{MC}\right)$, then $\mathrm{Con}\left(\mathrm{ZFC}+\omega_{1}-\mathrm{MC}+\mathrm{GCH}+\sim(\mathrm{R})\right)$ and $\mathrm{Con}\left(\mathrm{ZFC}+\omega_{1}-\mathrm{MC}+\sim \mathrm{GCH}+\sim(R)\right)$.

Proof. Suppose $M$ is a countable model of $\mathrm{ZFC}+\omega_{1}-\mathrm{MC}$. By [5], there is a generic extension $N$ of $M$ to a model of $\mathrm{ZFC}+\omega_{1}-\mathrm{MC}+\mathrm{GCH}$. By 2.3, N can be generically extended to a model of $\mathrm{ZFC}+\omega_{1}-\mathrm{MC}+\mathrm{GCH}+$ $\sim(R)$. Note that the algebra $B$ in 2.3 leaves uncountably many of the measurable cardinals measurable. To prove the second claim it suffices to note that by [7] $N$ can be so chosen that $N \neq \sim \mathrm{GCH}$. 


\section{A connection with generalized quantifiers}

3.1. We relate the axioms $(P)$ and $(R)$ to the equivalence of certain abstract logics. The above independence results can then be interpreted as reflecting the relative strengths of these logics. This connection with abstract logics also leads to problems of independence of certain weaker forms of $(P)$ and $(R)$.

3.2. The following result was proved in [12]: A model class is definable in $\Delta(L I)\left(\Delta(L R), \Delta\left(L^{I I}\right)\right)$ if and only if it is $\Delta_{1}(C d)-$ $\left(\Delta_{1}(\mathrm{Rg})-, \Delta_{1}(\mathrm{Pw})-\right.$, respectively) definable in set theory. For unexplained notation the reader is referred to [12] or [9]. The axiom ( $P$ ) is equivalent to saying that every $\Delta_{1}(\mathrm{Pw})$-predicate is $\Delta_{1}(\mathrm{Cd})$-definable. Hence

$$
(P) \leftrightarrow \Delta(L I)=\Delta\left(L^{I I}\right)
$$

Similarly,

$$
(R) \leftrightarrow \Delta(L I)=\Delta(L R)
$$

Combined with the results of Chapter 2 , this yields the consistency of

$$
\begin{aligned}
& \Delta(\mathrm{LI})=\Delta\left(\mathrm{L}^{\mathrm{II}}\right), \\
& \Delta(\mathrm{LI}) \neq \Delta\left(\mathrm{L}^{\mathrm{II}}\right)+\mathrm{GCH}, \\
& \Delta(\mathrm{LI}) \neq \Delta\left(\mathrm{L}^{\mathrm{II}}\right)+\sim \mathrm{GCH},
\end{aligned}
$$

relative to the consistency of $\mathrm{ZF}$, and also the consistency of

$$
\begin{aligned}
& \Delta(L I) \neq \Delta(L R)+\mathrm{GCH}, \\
& \Delta(L I) \neq \Delta(L R)+\sim \mathrm{GCH},
\end{aligned}
$$

relative to the consistency of $Z F C+\omega_{1}-M C$.

Using well-known preservation properties of the $\Delta$-operation one can prove that $(P)$ implies the following axioms: 
(P1) LI and $L^{I I}$ have the same Löwenheim numbers.

(P2) LI and $\mathrm{L}^{\mathrm{II}}$ have recursively isomorphic decision problems.

(P3) $\Delta(L I)$ and $L^{I I}$ have the same Hanf numbers.

3.3. P $\mathrm{r} \circ \mathrm{b} I \mathrm{e} \mathrm{m}$. (P) irmplies (P1), (P2) and (P3). Are there any other implications between these axioms?

3.4. A Boolean extension of $L$ in which the decision problem of LI is $\Delta_{3}^{1}$ was constructed in [11]. Thus $\sim(P 2)$ is consistent relative to $\operatorname{Con}(\mathrm{ZF})$. In [13] a Boolean extension was constructed in which the Hanf number of $\Delta(L I)$ is less than the Hanf number of $\Sigma(L I)$ (see [12] or [13] for a definition of $\Sigma$ ) and therefore less than the Hanf number of $L^{I I}$. Thus $\sim(P 3)$ is consistent relative to $\operatorname{Con}(Z F)$.

Axiom ( $P 1$ ) has a purely set-theoretical form. It was proved in [12] that the Löwenheim number of LI (LR, $L^{I I)}$ is $\sup \{\alpha \mid \alpha$ is $\Pi_{1}(\mathrm{Cd})-\left(\Pi_{1}(\mathrm{Rg})-, \Pi_{1}(\mathrm{Pw})-\right.$, respectively $)$ definable . For any set $D$ of set-theoretical formulae let us define (whenever possible):

$$
O(D)=\sup \{\alpha \mid \alpha \text { is definable by a formula of } D\} \text {. }
$$

Then

$$
\begin{aligned}
(\mathrm{P} 1) & \leftrightarrow \circ\left(\Pi_{1}(\mathrm{Cd})\right)=o\left(\Pi_{1}\left(\mathrm{Pw}_{\mathrm{w}}\right)\right) \\
\leftrightarrow & \circ\left(\Pi_{1}(\mathrm{Cd})\right)=o\left(\Pi_{2}\right) .
\end{aligned}
$$

Note that $\exp (\omega)$ is $\pi_{2}$-definable. Therefore

$$
(P 1) \rightarrow \exp (\omega)<o\left(\Pi_{1}(C d)\right) \text {. }
$$

In [11] we constructed a Boolean extension of $L$ in which $o\left(\Pi_{1}(C d)\right)<\exp (\omega)$. Thus $\sim(P 1)$ is consistent relative to Con $(Z F)$. This result is independently due to J. Stavi. For further and stronger results in this direction see [10]. There is also a consistency result for $\sim(P 1)+\mathrm{GCH}$ : In [14] we construct, starting with a proper class of 
measurable cardinals, a Boolean extension in which $\mathrm{GCH}$ holds and

$$
o\left(\Pi_{1}(C d)\right)<\text { the first measurable cardinal }<o\left(\Pi_{2}\right) .
$$

This raises the following problem:

3.5. P $\mathrm{r} \circ \mathrm{b} l \mathrm{e} \mathrm{m}$. Does $\mathrm{Con}(\mathrm{ZF})$ imply $\mathrm{Con}(\mathrm{ZFC}+\mathrm{GCH}+\sim(\mathrm{P} 1))$ ?

3.6. If the above analysis of $(P)$ is repeated for $(R)$, a more subtle situation emerges. As above, ( $R$ ) implies

(R1) LI and LR have the same Löwenheim numbers, that is, $o\left(\Pi_{1}(\mathrm{Cd})\right)=o\left(\Pi_{1}(\mathrm{Rg})\right)$.

(R2) LI and LR have recursively isomorphic decision problems.

(R3) $\Delta(L I)$ and $\Delta(L R)$ have the same Hanf numbers.

Problem 3.3 can be restated for $(R),(R 1),(R 2)$ and (R3). By elaborating the construction used for $\sim(R)$ we shall shortly construct models for $\sim(R 1)$ and $\sim(R 2)$.

3.7. $\mathrm{R}$ e $\mathrm{m}$ a $\mathrm{rk}$. If we use the results of [12], we get the following equivalences:

$$
\begin{aligned}
& (\mathrm{P} 1) \leftrightarrow \circ\left(\Delta_{1}(\mathrm{Cd})\right)=o\left(\Delta_{2}\right) . \\
& (\mathrm{P} 3) \leftrightarrow \circ\left(\Sigma_{1}(\mathrm{Cd})\right)=o\left(\Sigma_{2}\right) . \\
& (\mathrm{R} 1) \leftrightarrow \circ\left(\Delta_{1}(\mathrm{Cd})\right)=o\left(\Delta_{1}(\mathrm{Rg})\right) . \\
& (\mathrm{R} 2) \leftrightarrow \circ\left(\Sigma_{1}(\mathrm{Cd})\right)=o\left(\Sigma_{1}(\mathrm{Rg})\right) .
\end{aligned}
$$

3.8. One may also consider the axiom

$$
\text { (P)' Pw is } \Sigma_{1}(\mathrm{Rg})
$$

or, what amounts to the same: $\Delta(L R)=\Delta\left(L^{I I}\right)$. The resuits 1.7 and 1.8 carry over trivially to $(P)^{\prime}$ '. Analogously one may define (P1)', (P2)' and (P3)'. Apart form the result based on measurable cardinais, all consistency results of 3.4 carry over to these modified axioms. 


\section{Consistency results concerning (R1) and (R2)}

4.1. L e m m a (In GB + Global Choice). Suppose GCH. Let $\left\{\rho_{\alpha} \mid \alpha \in O_{n}\right\}$ be a sequence of measurable cardinals such that $\alpha<B$ implies $\alpha<\rho_{\alpha}<\rho_{\beta}$. There is an indexed family $\left\{B_{\alpha} \mid \alpha \in\right.$ On $\}$ of complete Boolean algebras such that for every $\alpha \in$ On the following hold:

(1) - (5) as in 2.2 .

(6) If $\mathrm{v}_{\alpha}^{\mathrm{B}} \vDash \check{k}$ is measurable, then $k=\rho_{\beta}$ for some $\beta \geq \alpha$.

Proof. The construction of $\left\{B_{\alpha} \mid \alpha<\omega_{1}\right\}$ in the proof of 2.2 can be continued over all ordinals. For a proof of (6) we refer to [8].

4.2. Th e o $\mathrm{e} \mathrm{m}$. If $\operatorname{Con}(\mathrm{ZFC}+$ there is a proper class of measurable cardinals $)$, then $\mathrm{Con}(\mathrm{ZFC}+\sim(\mathrm{R} 1)+$ there is a proper class of measurable cardinals).

Proof. Suppose $M$ is a countable model of $\mathrm{ZFC}+$ there is a proper class of measurable cardinals. By [5] $M$ has a generic extension $M^{\prime}$ to a model of $\mathrm{ZFC}+\mathrm{GCH}+$ there is a proper class of measurable cardinals. It is well-known (see [4]) that $M^{\prime}$ has a generic extension $N$ to a model of $\mathrm{GB}+$ Global Choice + GCH such that $M^{\prime}$ and $N$ have the same sets. Let $\left\{\rho_{\alpha} \mid \alpha \in O_{n}\right\}$ be an ascending well-ordering of the measurable cardinals of $N$. If there is an $\alpha$ such that $\alpha=\rho_{\alpha}$, we let $N_{1}$ be the restriction of $N$ to sets of rank less than $\alpha$, and otherwise $N_{1}=N$. Now $N_{1}$ is a model of $\mathrm{GB}+$ Global Choice + $\mathrm{GCH}+$ there is a sequence $\left\{\rho_{\alpha} \mid \alpha \in O_{n}\right\}$ of measurable cardinals such that $\alpha<\beta$ implies $\alpha<\rho_{\alpha}<\rho_{\beta}$. We shall work inside $N_{1}$ for a moment. Let $G:$ On $\rightarrow$ On such that $G\left(\rho_{\alpha}^{+}\right)=\rho_{\alpha}^{+++}$for $\alpha \in$ on and $G(\alpha)=0$ otherwise. Following Easton-forcing $([3])$, we consider sets of conditions p with the properties:

$$
\begin{aligned}
& p \subset \underset{\alpha}{\mathrm{p}}\{\alpha\} \times \omega_{\alpha} \times G\left(\omega_{\alpha}\right) \times 2, \\
& \forall B \forall \gamma_{1} \forall \gamma_{2}\left(\sim \left(<\beta, \gamma_{1}, \gamma_{2}, 0>\epsilon \mathrm{p} \wedge\right.\right. \\
& \left.\left.\quad<\beta, \gamma_{1}, \gamma_{2}, 1>\epsilon p\right)\right), \\
& \forall \beta \operatorname{card}\left(\left\{<\gamma_{1}, \gamma_{2}, i>\mid<\beta, \gamma_{1}, \gamma_{2}, i>\epsilon p\right\}\right)<\omega_{\beta} .
\end{aligned}
$$


Note that Lemma 35 of [3] still holds for this forcing. We modify the proof of Theorem 10 in [7] to prove that each $\rho_{\alpha}$ is still measurable in the generic extension $\mathrm{N}_{2}$ of $\mathrm{N}_{1}$ in this forcing. Suppose $F$ is a normal ultrafilter on $\rho_{\alpha}$ in $\mathrm{N}_{1}$ and $\mathrm{F}^{\prime}$ is the filter on $\rho_{\alpha}$ which is generated by $F$ in $N_{2}$. Suppose $u$ denotes a subset of $\rho_{\alpha}$ in $\mathbb{N}_{2}$. By Lemma 35 of [3] there is a condition $p(k)$ in the generic sequence and a set $\Pi$ such that (in the notation of [3])

(i) $\quad \mathrm{p} \in \mathrm{\pi} \rightarrow \mathrm{p} \subset \Gamma_{\rho_{\alpha}}$.

(ii) $\quad p^{(k)} \mid \vdash u \subset \rho_{\alpha}$.

(iii) If $\gamma<\rho_{\alpha}$ and $q \leq p^{(k)}$, then there is some $p \in \pi$ such that $p \cup p^{(k)}$ decides the statement $\Upsilon \in u$ and $p$ is compatible with $q$.

Note that $\operatorname{card}\left(\Gamma_{\rho_{\alpha}}\right)<\rho_{\alpha}$, as $\rho_{\alpha}$ is strongly inaccessible. Hence also $\operatorname{card}(\Pi)<\rho_{\alpha}^{\alpha}$. We shall use the properties (i) - (iii) to prove that $\mathrm{N}_{2}$ satisfies $u \in F^{\prime} v \rho_{\alpha}-u \in F^{\prime}$. If $q \leq p^{(k)}$ and $q \mid F \underline{\beta} \in u$, then by ( $i i i)$ there is some $p_{0} \in \pi$, compatible with $q$, such that $p_{0} \cup p^{(k)}$ decides $\underline{B} \in u$. If $p_{0} \cup p^{(k)} \mid+\underline{B} \notin u$, then $p_{0} \cup q \mid-\underline{B} \notin u$, which contradicts $q \mid F \underline{\beta} \in u$. Therefore $\mathrm{p}_{0} \cup \mathrm{p}^{(\mathrm{k})} \mid-\underline{B} \in \mathrm{u}$. Conversely, if $\underset{\mathrm{p}}{\mathrm{p}} \cup \mathrm{p}_{(\mathrm{k})} \mid \leftarrow \underline{\beta} \in \mathrm{u}$ for some $p_{0} \in \Pi$ then there is a $q \quad\left(=p_{0} \cup p^{(k)}\right) \leq p^{(r)}$ such that $q \mid F$ $\underline{\beta} \in$ L. Thus we have proved

$$
\begin{aligned}
& \left\{\beta<\rho_{\alpha} \mid \exists q \leq p^{(k)}(q \mid \vdash \underline{\beta} \in u)\right\}= \\
& \bigcup_{p_{0} \in \Pi}\left\{\beta<\rho_{\alpha}\left|\left(p_{o} \cup p^{(k)}\right)\right| \vdash \underline{B} \in u\right\} .
\end{aligned}
$$

Let us denote this set by $X$. If $X$ is not in $F$, then $\left\{\beta<\rho_{\alpha}\left|p^{(k)}\right| \vdash \underline{\beta} \notin u\right\} \in F$, whence $N_{2} \models \rho_{\alpha}-u \in F^{\prime}$. Suppose then $X$ is in $F$. By completeness there is a $p_{0} \in \Pi$ such that $Y=$ $\left\{\beta<\rho_{\alpha}\left|\left(p_{0} \cup p^{(k)}\right)\right| \vdash \underline{\beta} \in u\right\} \in F$. Note that $Y$ is in $N_{1}$ and $\mathrm{p}^{(\mathrm{k})} \mid L^{\prime} \mathrm{y} \subset \mathrm{u}$. Hence $\mathrm{N}_{2} \models u \in \mathrm{F}^{\prime}$. We have proved that $\mathrm{F}^{\prime}$ is an ultrafilter. The $\rho_{\alpha}$-completeness of $F^{\prime}$ is proved similarly. Thus 
$\rho_{\alpha}$ is measurable in $\mathrm{N}_{2}$.

We shall work now in $N_{2}$. Let $\left\{B_{\alpha} \mid \alpha \in\right.$ On $\}$ be as in 4.1. To finish the proof we construct an $\alpha \in$ On such that

$$
\text { (*) } \quad \mathrm{v}^{\mathrm{B}}{ }_{\alpha} \in \sim(\mathrm{R} 1) \text {. }
$$

Let $\left\{\varphi_{n}(x) \mid n<\omega\right\}$ be a recursive enumeration of all $\pi_{1}(C d)$-formulae with one free variable. Let $M(n)$ be the $\Sigma_{1}(C d)$-predicate

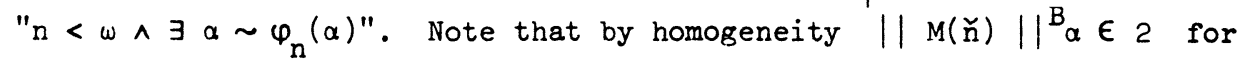
every $\alpha \in$ On and $n \in \omega$. Let $\alpha_{o_{B}}=0$. If $\alpha_{n}$ is defined and there is a $B>\alpha_{n}$ such that $\|M(\check{n})\|{ }_{B} B_{B}=1$, let $\alpha_{n+1}$ be the least such $\beta$, and otherwise $\alpha_{n+1}=\alpha_{n}$. Let $\beta=\sup \left\{\alpha_{n} \mid n<\omega\right\}$. Let $k$ be a cardinal such that $\mathrm{V}_{B} \vDash \forall n<\check{\omega}\left(M(n) \rightarrow \exists \alpha<\check{\kappa}\left(\sim \varphi_{n}(\alpha)\right)\right)$. Let $\alpha \in$ On such that $\rho_{\alpha}>k$. We claim that $\alpha$ satisfies $(*)$, and, indeed, that

$$
v^{B}=o\left(\Pi_{1}(C d)\right) \leq \check{k}<o\left(\Pi_{1}(R g)\right)
$$

Suppose $n<\omega$ and $\Phi_{n}(x)$ defines the ordinal $\check{\gamma}$ in $v^{B}$. Then $\|M(\check{n})\|_{\alpha}^{B}=1$, whence, by construction, $\|M(\check{n})\|_{B}^{B}=1$. Hence for some $\delta<k, V^{B}=\sim_{n}(\check{\delta}) \wedge \check{\gamma}<\check{k}$. Thus $V_{\alpha}^{B} \models o\left(\Pi_{1}(C d)\right) \leq \check{k}$. To prove $v_{\alpha}^{B} \models \tilde{k}<o\left(\Pi_{1}(R g)\right)$ it suffices to note that $\check{\rho}_{\alpha}^{+}$is $\Pi_{1}(\mathrm{Rg})$-definable in $\mathrm{v}_{\alpha}^{\mathrm{B}}$. In fact, it is true in $\mathrm{v}_{\alpha}^{\mathrm{B}}$ that $\gamma>\rho_{\alpha}^{+}$ if and only if there is a regular cardinal $\lambda$ such that $\lambda^{+} \leq \gamma$ and $\exp \left(\lambda^{+}\right) \geq \lambda^{+++}$. $\sim(\mathrm{R} 2))$.

4.3. The or e m. If $\operatorname{Con}\left(Z F C+\omega_{1}-M C\right)$, then $\operatorname{Con}\left(Z F C+\omega_{1}-M C+\right.$

Proof. The proof resembles that of the previous theorem, and some of the details can be omitted. Let us start with a model $\mathrm{N}_{1}$ of $G B+\omega_{1}-M C+G C H$. Let $\left\{\rho_{\alpha} \mid \alpha<\omega_{1}\right\}$ be an ascending sequence of measurable cardinals in $N_{1}$. We shall work in $N_{1}$. Let $\left\{a_{\alpha} \mid \alpha<\omega_{1}\right\}$ be a sequence of infinite almost disjoint subsets of $\omega$. Let $\left\{D_{\alpha} \mid \alpha<\omega_{1}\right\}$ be an ascending sequence of homogeneous cardinals preserving Boolean algebras such that $\rho_{\beta}$ is singular in $v^{D_{\alpha}}$ if and only if $\beta<\alpha$, and $\exp \left(\omega_{\gamma}\right) \geq \omega_{\gamma+2}$ holds in $v_{\alpha}^{D}$ if and only if $\gamma=n_{\beta}+n+1$ where $n \in a_{B}$ and $B \leq \alpha$. We claim that (R2) fails 
in $v^{D}$ for some $\alpha<\omega_{1}$. Suppose the contrary. Let $\left\{\varphi_{n} \mid n<\omega\right\}$ be a primitive recursive enumeration of all LR-sentences and $\left\{\psi_{n} \mid n<\omega\right\}$ a similar list of all LI-sentences. By a simple pigeonhole argument there are $\alpha, \beta$ and a (description of a) recursive function $f$ such that $\alpha<\beta<\omega_{1}$ and the sentence $\forall \mathrm{n}\left(\varphi_{\mathrm{n}}\right.$ has a model if and only if $\psi_{f(n)}$ has) is true both in $v_{\alpha}^{D_{\alpha}}$ and in $v^{D}$. Let for any $\mathrm{n}<\omega, \varphi_{\mathrm{g}(\mathrm{n})}$ be the LR-sentence which says that $\exp \left(\omega_{\delta}\right)>\omega_{\delta+2}$ holds for some $\delta=u+n+1$, where $u$ is an inaccessible cardinal. To derive finally a contradiction, we prove that $a_{\alpha} \subset a_{\beta}$. So let $n \in a_{\alpha}$. Then $\varphi_{g(n)}$ has a model in $v^{D}$. Therefore, $\psi_{f(g(n))}$ has a model in $v^{D}$. As cardinals are preserved in our extensions, $\psi_{f}(g(n))$ has a model in $v^{D_{B}}$, whence $\varphi_{g(n)}$ has a model in $v^{D_{B}}$. By construction, this implies $n \in a_{B}$. This ends the proof of $a_{\alpha} \subset a_{\beta}$. The arising contradiction shows that (R2) fails in some $v^{D}$. 4.4. In fact, the above two constructions can be put together to yield a model in which $\sim(R 1)$ and $\sim(R 2)$ hold simultaneously. A similar, but, in a sense, reversed argument can be used to prove the consistency of $\sim(\mathrm{R} 3)$ relative to the consistency of a proper class of measurable cardinals. We omit the details of this construction. It remains an open problem whether one can have $\sim(R 1), \sim(R 2)$ and $\sim(R 3)$ simultaneously.

\section{References}

[1] Devlin, K., and R. Jensen : Marginalia to a theorem of Silver. - Logic Conference Kiel 197'4, Lecture Notes in Mathematics 499, Springer-Verlag, Berlin - Heidelberg - New York, 1975, pp. 115-142.

[2] Drake, F.: Set theory. - North-Holland Publishing Company, Amsterdam - London, 1974.

[3] Easton, W.: Powers of regular cardinals. - Ann. Math. Logic 1, 1970, pp. 139-178.

[4] Felgner, U.: Comparison of the axioms of local and universal choice. - Fund. Math. 71, 1971, pp. 43-62.

[5] Jensen, R.: Measurable cardinals and the GCH. - Proceedings of Symposia in Pure Mathematics XIII part 2, Axiomatic set theory, Amer. Math. Soc., Providence, Rhode Island, 1974, 
pp. $175-178$.

[6] Kunen, K.: Some applications of iterated ultrapowers in set theory. - Ann. Math. Logic 1, 1970, pp. 179-227.

[7] Lévy, A., and R. Solovay : Measurable cardinals and the continuum hypothesis. - Israel J. Math. 5, 1967, pp. 234-248.

[8] Magidor, M.: How large is the first strongly compact cardinal? - Ann. Math. Logic 10, 1976, pp. 33-57.

[9] Makowsky, J.A., S. Shelah, and J. Stavi : $\Delta$-logics and generalized quantifiers. - Ann. Math. Logic 10, 1976, pp. 155-192.

[10] Stavi, J., and J. Väänänen : Reflection principles for the continuum. - In preparation.

[11] Väänänen, J.: Boolean valued models and generalized quantifiers. - To appear.

[12] Väänänen, J.: Abstract logic and set theory. I. Definability. - To appear.

[13] Väänänen, J.: $\Delta$-extensions and Hanf numbers. - To appear.

[14] Väänänen, J.: Abstract logic and set theory. II. Large cardinals. - To appear.

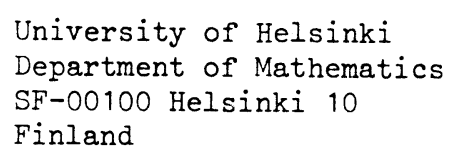

